\title{
Teneurs en 180 et concentration saline d'eaux paraliques et continentales égyptiennes $0^{18}$ content and sait concentration of lagoonal and continental egyptian waters
}

\author{
C. Jusserand, A. W. el S. Ibrahim, O. Guelorget et J. P. Perthuisot
}

Volume 1, numéro 3, 1988

URI : https://id.erudit.org/iderudit/705013ar

DOI : https://doi.org/10.7202/705013ar

Aller au sommaire du numéro

\section{Éditeur(s)}

Université du Québec - INRS-Eau, Terre et Environnement (INRS-ETE)

\section{ISSN}

0992-7158 (imprimé)

1718-8598 (numérique)

\section{Découvrir la revue}

Citer cet article

Jusserand, C., Ibrahim, A. W. e. S., Guelorget, O. \& Perthuisot, J. P. (1988). Teneurs en 180 et concentration saline d'eaux paraliques et continentales égyptiennes. Revue des sciences de l'eau / Journal of Water Science, 1(3), 277-301. https://doi.org/10.7202/705013ar

\section{Résumé de l'article}

Plusieurs corps d'eau lagunaires et continentaux égyptiens sont comparés quant à l'abondance en oxygène-18 et la concentration saline (ici appréciée par la teneur en $\mathrm{Cl}^{-}$) de leurs eaux superficielles.

En Egypte, les eaux continentales de surface proviennent toutes du système nilotique dont les eaux, après leur longue traversée du grand Desert Oriental, se trouvent très évaporées et fortement enrichies en oxygène-18 à leur arrivée dans le delta.

Dans les bassins alimentés uniquement par la mer (lagunes de Mer Rouge et du Golfe de Suez) soit par l'eau du Nil (Lac du Fayoum), les deux paramètres considérés augmentent conjointement depuis les zones d'alimentation vers les reculées marginales selon le déplacement des eaux.

Dans les bassins à alimentation mixte, (Partie terminale du delta, lac Temsah sur le Canal de Suez), les eaux les plus diluées sont aussi les plus riches en isotopes lourds.

Ainsi, dans le contexte climatique et géographique très particulierde l'Egypte, il est possible de reconnaître les eaux continentales, marines ou paraliques à partir des deux paramètres étudiés mais non à partir d'un seul, notamment la teneur en oxygène-18. 


\title{
Teneurs en ${ }^{18} 0$ et concentration saline d'eaux paraliques et continentales égyptiennes
}

\author{
$0^{18}$ content and salt concentration \\ of lagoonal and continental egyptian waters
}

C. JUSSERAND (1)*, A. IBRAHIII W. el S. (2), 0. GUELORGET [3], et J.P. PERTHUISOT (4)

RÉSUMÉ

Plusieurs corps d'eau lagunaires et continentaux égyptiens sont comparés quant à l'abondance en oxygène-18 et la concentration saline (ici appréciée par la teneur en $\mathrm{Cl}^{-}$) de leurs eaux superficielles.

En Egypte, les eaux continentales de surface proviennent toutes du système nilotique dont les eaux, après leur longue traversée du Grand Desert Oriental, se trouvent très évaporées et fortement enrichies en oxygène-18 à leur arrivée dans le delta.

Dans les bassins alimentés uniquement par la mer (lagunes de Mer Rouge et du Golfe de Suez) soit par l'eau du Nil (Lac du Fayoum), les deux paramètres considérés augmentent conjointement depuis les zones d'alimentation vers les reculées marginales selon le déplacement des eaux.

Dans les bassins à alimentation mixte, (Partie terminale du delta, Lac remsah sur le Canal de Suez), les eaux les plus diluées sont aussi tes plus riches en isotopes lourds.

(1)* : Département de Géologie Dynamique, Université P. et M. Curie, 4 place Jussieu, F-75005 Paris.

(2) : Université d'El Arich, El Arich, Egypte.

(3) : Laboratoire d'Hydrogiologie Marïne, USTL, Place E. Batailion, F-34000 Montpellier.

(4) : Laboratoire de Biogéologie et Biostratigraphie, Université de Nantes, 2 rue de la Houssinière, F-44000 Nantes. 
Ainsi, dans le contexte climatique et géographique très particulierde l'Egypte, il est possible de reconnaitre les eaux continentales, marines ou paraliques à partir des deux paramètres étudiés mais non à partir d'un seul, notamment la teneur en oxygène-18.

Mots clés : oxygène-18, salinitè, paralique, continental, Egypte, actuel.

The aim of this paper is to study some aspects of the geochemical behaviour $\left({ }^{18} 0,\left(\mathrm{Cl}^{-}\right)\right)$of waters of some basins in Egypt. Several types of basin are studied (figure 1).

1) Basin with marine seawater input only, either wide open to the Red sea such as Zeit Bay $\left(27^{\circ} 45^{\prime} \mathrm{N}, 33^{\circ} 25^{\prime} \mathrm{E}\right)$, relatively open such as Guesmah Lagoon $\left(27^{\circ} 40^{\prime} \mathrm{N}, 33^{\circ} 30^{\prime} \mathrm{E}\right)$ or indirectly linked to the sea through a coastal sand bar such as the pools of sebkha Melaha $\left(28^{\circ} 10^{\prime} \mathrm{N}, 33^{\circ} 10^{\prime} \mathrm{E}\right)$.

2) Basin supplied by continental water without outflow such as Fayoum Lake ( $=$ Birket Karoum) $\left(29^{\circ} 25^{\prime} \mathrm{N}, 30^{\circ} 30^{\prime} \mathrm{E}\right)$ which receives fresh waters from a Nile diversion.

3) Water bodies with a mixed water input (continental and marine waters) such as the Nile delta and Temsah Lake (= Ismaila Lake), $\left(30^{\circ} 25^{\prime} \mathrm{N}, 31^{\circ} 30^{\prime} \mathrm{E}\right)$, the latter being on the Suez Canal and receiving waters from Mediterranean Sea and from the eastern branch of the Nile.

Local means climatic parameter are those of Cairo, Alexandria, Queseir and Louxor (table 1).

RESULTS AND DISCUSSION

1 - Nile delta (figure 2 and 3 )

Sampling period was performed in August 1984.

- At the beginning of the Nile delta the water at Beni-Suez (station 14) presents a low chloride concentration 10.1 to $0.2 \mathrm{~g}^{-1}$ ) and a relatively high ${ }^{18} 0$ content ( $3 \%$ ) due to evaporation of the Nile water during its downstream course from the Aswan High Dam which collects waters in the upper part of the Nile watershed.

- The content of both $\left(\mathrm{Cl}^{-}\right)$and ${ }^{18} 0$ slightly increases in the delta region in relation to the anastomosis of branches of the $\mathrm{Nile}$ due to an intensive irrigation network.

- In the coastal region, due to mixing with sea water or/and brackish water, the isotope content decreases while the chloride concentration increases.

2 - Temsah or Ismaila Lake

Two periods of sampling: March and August 1984. The general trend is a decrease of ${ }^{18} \mathrm{O}$ and an increase of $\left(\mathrm{Cl}^{-}\right)$from the Nile waters to the central part of the Lake, and an increase of both ${ }^{18}{ }_{0}$ and $\left(\mathrm{CL}^{-}\right)$at the fringes of the lake, due to evaporation. 


\section{3 - Coastal basins from the Red Sea}

Zeit Bay and Guesmah Lagoon. Two sampling periods : March and August 1984. The isotope content and chloride concentration act in parallel and depend principally on the movement of water bodies. For the surface waters wind velocity and direction play a major role in the spatial distribution of the chlorinity and isotope content. A slight enrichment is noted during the summer period.

\section{4 - Pools in Sebkha Melaha}

The isotope composition and chloride content of the ponds water fed by sea water through the sand salt bar increased during the summer as it is the case in the first stages of evaporation of salt pans (PIERRE and FONTES, 1982).

. 5 - Fayoun Lake : Birket Karoum

Two sampling periods : March and August 1984. Because of a lack of sufficient data (salinity and isotopic composition of the input, water flux of the incoming water, local values of the relative humidity and temperature of the atmosphere, isotopic composition of the water vapour ...) a water mass balance coupled with a chloride and isotopic mass balance was not possible. Nevertheless, with our data measured $\left({ }^{18} \mathrm{O},\left(\mathrm{Cl}^{-}\right)\right.$lake (evels), some estimations were made of the temperature, evaporation, wind velocities (from meteorological tables) and the isotopic composition of the input : $\delta_{A}$ in the lake (SIMPSON et al., 1987), and if we assume that the lake presents a long term dynamic equilibrium, it is possible to estimate with reasonable accuracy that : i) the total isotopic enrichment factor $(\varepsilon)$ is between $8 \%$ and $24 \%$ (figure 12) : $\varepsilon=\varepsilon_{e}+\varepsilon_{k}$, with $\varepsilon_{e}=$ equilibrium isotopic enrichment factor $; \varepsilon_{k}=$ kinetic isotopic enrichment factor. $i i)$ the salt content of the incoming water $\delta_{A}$ is $\approx 20 \mathrm{~g} \mathrm{t}^{-1}$. The principal consequence is that the important loss of water observed between the sampling periods ( $1.5 \mathrm{~m}$ difference in water level) was not due to evaporation only, but more by seepage from the bottom of the lake $\left(\approx 56010^{6} \mathrm{~m}^{3}\right.$ for a total volume of $\left.\approx 80010^{6} \mathrm{~m}^{3}\right)$.

\section{CONCLUSIONS}

1) On a diagram $\delta^{18} \mathrm{O}-\mathrm{Cl}^{-}$(figure 13 and 14) where all the data for the Egyptian basins studied are represented, the similarity in the geochemical behaviour of the basins with a single input, is noted; the relation between isotopic composition and chloride content is approximatively logarithmic. In the case of a mixed input basin (Temsah Lake) two branches on the diagram are distinct; the first branch shows the effect of mixing in the west part of the lake, between evaporated Nile water and water from the Mediterranean Sea. The shape of this branch is very similar to that of the Nile delta: the second branch represents evaporation in the east part of the lake (figure 13 and 14).

2) In the case of a semi-arid environment, this study shows that with. two simple parameters only, such as isotopic composition and the chloride content of waters, it is possible to distinguish the marine, paralic and continental domain (figure 15). 
L'Egypte possède, à la surface de notre planète, une situation géographique très originale qui explique d'ailleuxs pour une bonne part son évolution historique. Située au coeur de la ceinture aride de 1 'hêmisphère nord, elle ne reçoit sur son sol, hormis dans les parties les plus septentrionales, qu'une faible quantité d'eau météorique d'ailleurs très rapidement évaporée dès qu'elle a touché le sol (tableau 1). Le réseau hydrographique pérenne, celui du Nil, ne charrie que des eaux venues des régions arrosées tropicales et équatoriales, après une course de plus de $2000 \mathrm{~km}$ pendant laquelle elles ne sont soumises qu'à l'évaporation.

Il était intéressant d'étudier et de comparer des bassins alimentés soit seulement par la mer, soit seulement par l'eau du Nil, soit enfin de façon mixte, pour essayer de caractériser le comportement géochimique des eaux paraliques et continentales dans un tel contexte, notamment quant aux relations entre l'abondance en isotopes lourds (ici, l'oxygène-18) et la concentration saline (ici appréciée par la concentration en $\mathrm{Cl}^{-}$, ion le plus abondant et conservatif).

Les sites étudiés (figure 1) ont fait l'objet de deux campagnes de prélèvements, I'une en hiver (janvier ou mars 1984), l'autre en été (aolat 1984). Au cours de celles-ci ont été échantillonnés respectivement 15 et 12 points dans la lagune de Guemsah, 8 et 6 points dans la Baie de Zeit, 8 et 8 points dans le Lac Temsah, 13 et 8 points dans la Birket Karoun, 4 et 2 points dans les "Flaques" de la Sebkha Melaha. En outre, l'eau du Nil a été prélevée en 14 stations depuis la mer jusqu'en amont de la ville du Caire.

\section{1 - LES BASSINS, LEURS CARACTÉRISTIQUES CLIMATIQUES}

1 - La lagune de Guemsah $\left(27^{\circ} 40^{\circ} \mathrm{N}, 33^{\circ} 30^{\circ} \mathrm{E}\right)$

La lagune de Guemsah couvre une superficie de $8 \mathrm{~km}^{2}$ envixon pour une profondeur atteignant $15 \mathrm{~m}$ dans une dépression centrale flanquée de platiers peu profonds. Le bassin communique avec le domaine marin par trois passes d'inégale largeur seuillées par des dépóts sédimentaires à 1,5 m de profondeux (IBRAHIM, 1986 ; IBRAHIM et al., 1985).

La circulation des eaux dans la lagune est sous la dépendance essentielle du vent qui souffle le plus souvent du secteur $N$, avec une vitesse moyenne de l'ordre de $5 \mathrm{~ms}^{-1}$. Les eaux en provenance de la mer se dirigent vers l'autre extrémité du bassin puis suivent les platiers en sens inverse de telle soxte que les eaux les plus évoluées, c'est-àdire les plus confinées, se trouvent sur ceux-ci.

Les caractéristiques climatiques locales se rapprochent de celle de la station de Quseir (tableau 1) située plus au sud et sont valables pour la Baie de Zeit et pour les "Flaques" de la Sebkha Melaha. On peut estimer que la moyenne mensuelle des températures maximales oscille entre $34{ }^{\circ} \mathrm{C}$ (aout) et $22{ }^{\circ} \mathrm{C}$ (janvier), celle des temperatures minimales entre $27^{\circ} \mathrm{C}$ (aout) et $14^{\circ} \mathrm{C}$ (janvier).

Les précipitations sont pratiquement inexistantes avec une moyenne annuelle sûrement inféxieure à $10 \mathrm{~mm}$, alors que l'évaporation potentielle est élevée, supérieure à $12 \mathrm{~mm} \cdot \mathrm{j}^{-1}$ en moyenne. 


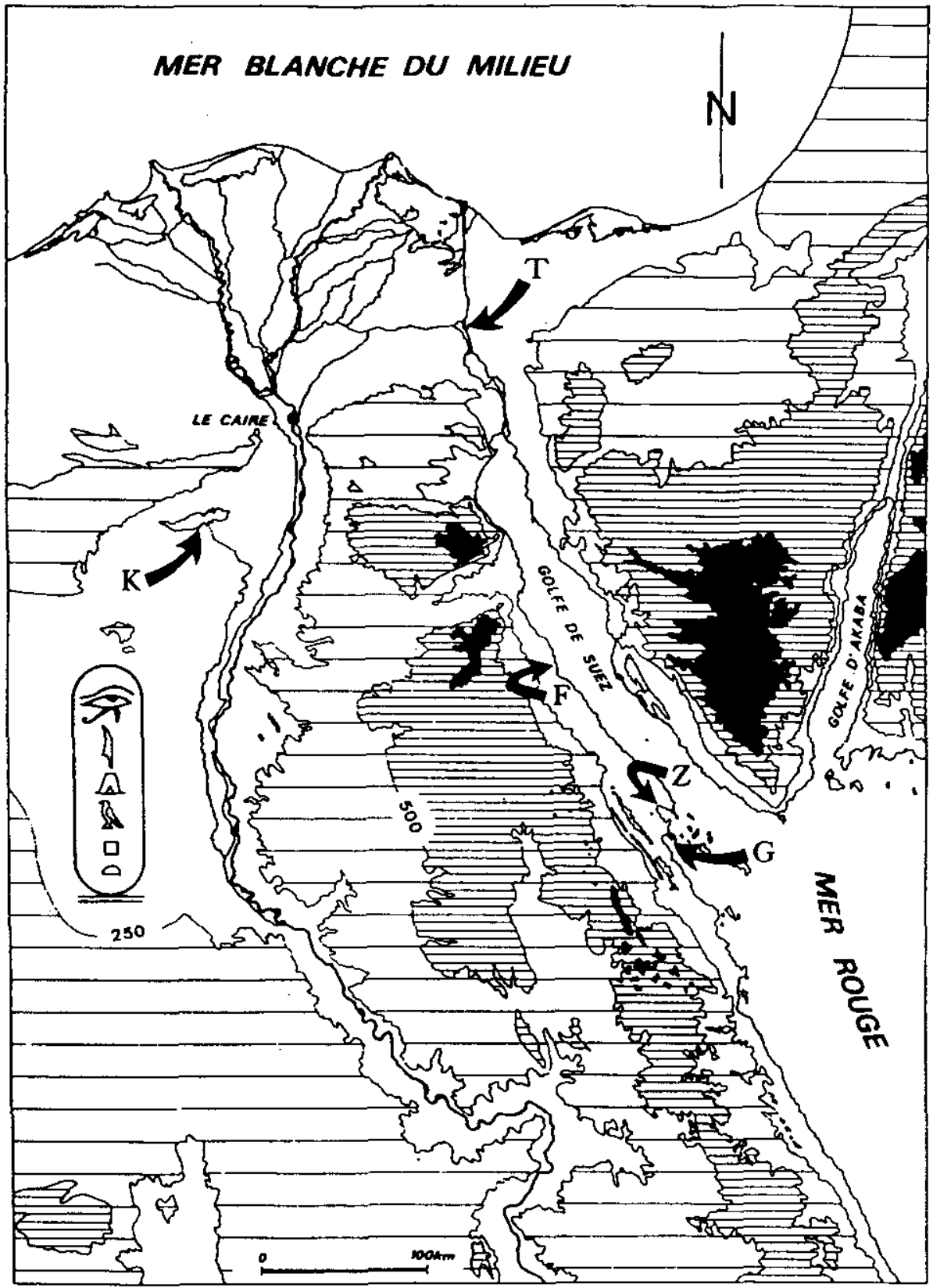

Figure 1.- Localisation des sites étudiés. G : Lagune de Guensah : Z : Baie de Zeit ; F : "Flaques" de la Sebkha Melaha ;

$T$ : Lac Temsah ; K : Birket Karoun (Lac du Fayoum).

Figure 1.- Situation of studied areas : $G$ : Gemsah Lagoon; $Z$ : Zeit Bay ; $F$ : Sabkha Malaha ponds ; $T$ : Temsah Lake; $K$ : Birket Qamon (Fayum Lake).

Les zones en noir de la carte correspondent aux régions d'altitude supérieures à $1000 \mathrm{~m}$.

Black areas : regions above $1000 \mathrm{~m}$. 


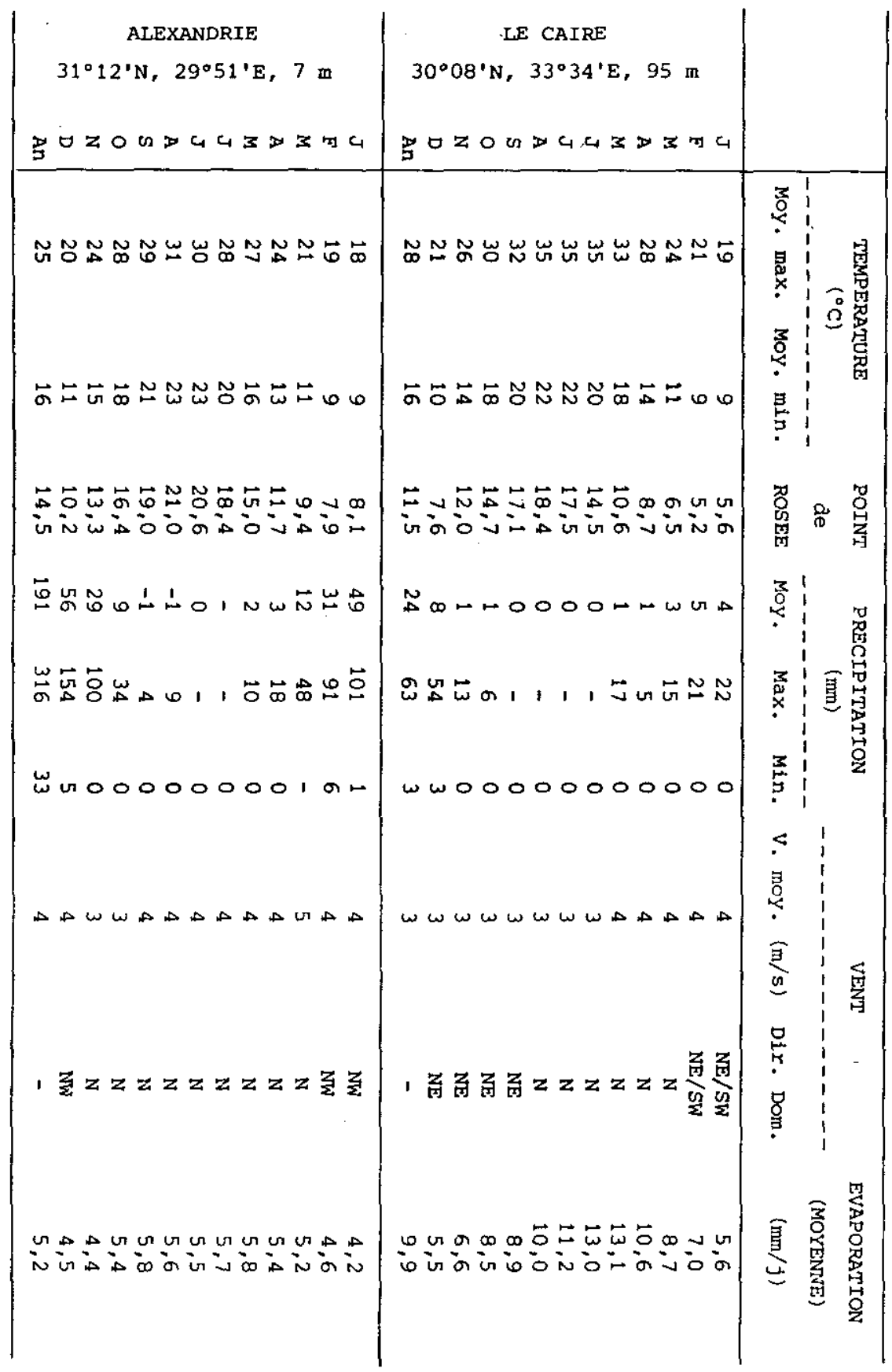




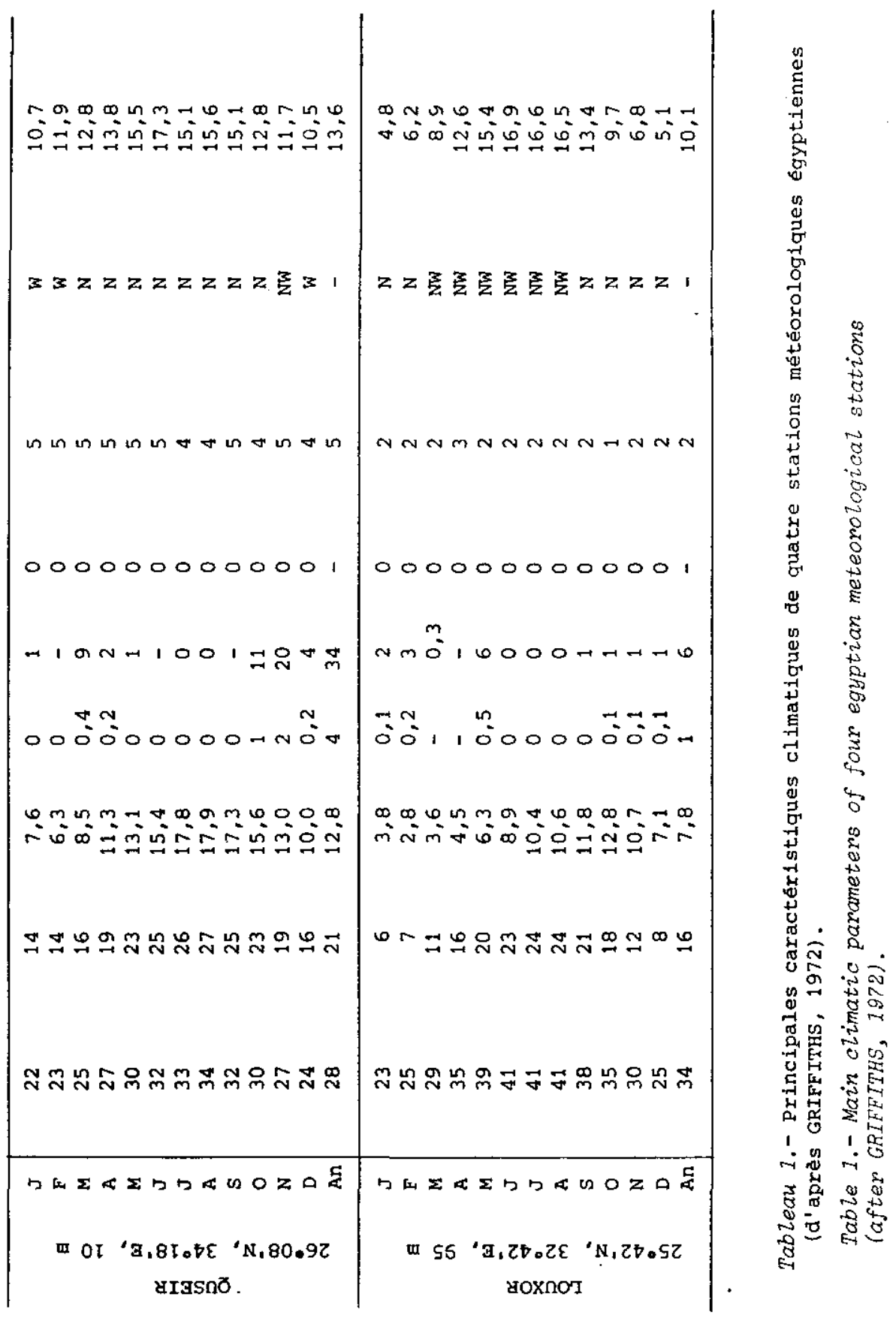


Dans le bassin, la température de l'eau de surface est plus variable sur les platìers latéraux (minimum enregistré : $13,2{ }^{\circ} \mathrm{C}$ en janvier 1983 ; maximum enregistré : $32{ }^{\circ} \mathrm{C}$ en septembre 1982 ) que dans le centre du bassin où elle oscille faiblement autour de $20^{\circ} \mathrm{C}$ (IBRAHIM, 1986).

2 - La Baie de Zeit $\left(27^{\circ} 45^{\prime} \mathrm{N}, 33^{\circ} 25^{\prime} \mathrm{E}\right)$

La Baie de Zeit couvre une superficie d'environ $70 \mathrm{~km}^{2}$. De forme grossièrement quadrangulaire, elle communique avec la mer ouverte par un dêtroit de $3 \mathrm{~km}$ de large seuillé à une profondeur de 7 ou $8 \mathrm{~m}$.

Comme pour la lagune de Guemsah, la circulation des eaux dans le bassin est très largement dépendante du régime des vents qui, sur cette vaste surface, sont encore plus actifs qu'à Guemsah et lèvent le plus souvent une mer assez dure. Le bassin dans son ensemble est moins confiné que la lagune de Guemsah et les eavx les plus évoluées se trouvent à sa marge méridionale.

3 - Les "Flaques" de la Sebkha Melaha $\left(28^{\circ}, 10^{\prime} \mathrm{N}, 30^{\circ}, 10^{\circ} \mathrm{E}\right)$

La Sebkha Melaha est un ancien bassin lagunaire séparé de la mer par un cordon littoral récent (IBRAHIM, 1986). Aujourd'hui asséché, il est comblé de sêdiments détritiques et évaporitiques. Il reste néanmoins alimenté par la mer en sous-écoulement à travers le cordon. Localement. cette alimentation est responsable de l'existence de flaques dont les saumures se perdent dans le remplissage sédimentaire et en rechargent la nappe. Un petit groupe d'entre elles a été étudié : elles contiennent des saumures dont la concentration ionique peut dêpasser $150 \mathrm{~g} \cdot 1^{1}$ en été et sont colonisées par une flore de Cyanophycées qui alimentent une faune de Gastéropodes paraliques (Potamides conicus) et de petits poissons du genre Aphanius.

\section{4 - Le lac Temsah ou Lac d'Ismailia $\left(30^{\circ} 35^{\prime} \mathrm{N}, 32^{\circ} 30^{\prime} \mathrm{E}\right)$}

Le lac Temsah est situé sur le canal de Suez approximativement â midistance entre Port Sald et Suez. Sa surface est de $12 \mathrm{~km}^{2}$ environ et sa profondeur atteint $20 \mathrm{~m}$ dans le chenal dragué qui occupe sa partie axiale.

Il est alimenté par un flux continu d'eau de mer en provenance de la Méditerranée comme en atteste la composition spécifique de la faune et de la flore, et par le bras le plus oriental du delta du Nil) qui se déverse dans la partie ouest du bassin. Cette alimentation mixte en est la principale originalité.

Le climat peut être considéré comme intermédiaire entre celui de Quseir et"celui d'Alexandrie (tableau 1) avec cependant une évaporation moyenne plus élevée que la moyenne entre ces deux stations cax l'influence de l'air marin est très atténuée : une moyenne de $10 \mathrm{~mm} . j^{-1}$ paxalt un chiffre raisonnable. La température moyenne du corps d'eau varie en fonction des saisons : autour de $18^{\circ} \mathrm{C}$ en hiver, elle dépasse $28^{\circ} \mathrm{C}$ en été (IBRAHIM, 1986). 


\section{5 - La Birket Karoun ou Lac du Fayoum $\left(29^{\circ} 25^{\circ} \mathrm{N}, 30^{\circ} 30^{\circ} \mathrm{E}\right)$}

La Birket Karoun occupe l'ombilic de la vaste dépression du Fayoum (1 $700 \mathrm{~km}^{2}$ ) et est alimentée par les seules eaux du systẻme nilotique (nappe phreatique des alluvions, lacis de chenaux d'irrigation issus du Bahr Youssef, diffluent du Nil).

Le bassin lui-même est allongé Est-Ouest sur environ $40 \mathrm{~km}$ et, pour une cote du plan d'eau de - $45 \mathrm{~m}$, il occupe une surface de $214 \mathrm{~km}^{2}$ et un volume d'environ $810.10^{6} \mathrm{~m}^{3}$. I'alimentation du bassin par les eaux de surface s'effectue principalement sur sa rive méridionale.

La circulation des eaux dans la Birket semble, comme pour les bassins précédents̀, sous la dépendance essentielle des vents du secteur $\mathrm{N}$ dominants (IBRAHIM, 1986).

Il n'existe pas de donnée directe sur le climat de la dépression du Fayoum, cependant, en interpolant entre les paramètres climatiques du Caire et ceux de Louxor, on peut avoir une bonne idée des conditions climatiques qui y règnent (tableau 1). La moyenne annuelle des maxima de la température est de $l^{\prime}$ 'ordre de $30^{\circ} \mathrm{C}$, celle des minima de $16^{\circ} \mathrm{C}$. La pluviométrie annuelle est très faible, de l'ordre de $20 \mathrm{~mm}$ par an en moyenne mais la répartition des pluies est très irrégulière et certaines années peuvent etre totalement sèches. L'évaporation annuelle sur eau pure et sur bac depasse à coup sar $3500 \mathrm{~mm}$ par an (moyenne du Caire : $9,9 \mathrm{~mm} . \mathrm{j}^{-1}$; moyenne à Louxor : $10,1 \mathrm{~mm} . \mathrm{j}^{-1}$ ). En tenant compte de l'effet de tallle (OMAR et EL BAKRY, 1987), un coefficient de 0,55 a 0,6 peut etre affecté aux données sur bac en climat aride : la valeur de l'évaporation moyenne annuelle réelle est donc voisine de $2150 \mathrm{~mm}$.

Les vents sont orientés du secteur $\mathrm{Nà} \mathrm{NW}$, avec une vitesse moyenne de $l^{\prime}$ ordre de $3 \mathrm{~ms}^{-1}$, sauf éventuellement en janvier et février, période au cours de laquelle on observe des vents du SW au Caire. C'est, au total, un climat extrêmement aride et seules les eaux du Nil rendent la région vivable.

Enfin, une des caractéristiques remarquables de la Birket Karoun est qu'elle contient une flore et une faune essentiellement constituée d'espèces paraliques (lagunaires) qui vivent habituellement dans les bassins en connexion avec la mer (IBRAHIM, 1986 ; PERTHUISOT et GUELORGET, $1987 a$ et b) ou ayant conserve une"mémoire'ionique de son origine marine partielle, comme c'est le cas pour la Mer Caspienne.

Ceci pose évidemment le problème d'une éventuelle alimentation marine de la Birket Karoun, actuellement ou dans un passé plus ou moins lointain : nous verrons ci-après qu'une telle éventualité est exclue actuellement et que si elle s'est produite, il ne peut subsister dans le bassin qu'une infime quantite d'eau d'oxigine marine. 


\section{2 - LE COMPORTEMENT ISOTOPIQUE ET GÉOCHIMIQUE DES BASSINS (EAUX DE SURFACE)}

\section{1 - Mẽthode}

$L^{\prime}$ 'abondance en oxygène-18 des eaux a été des saumures mesurée par spectrométrie de masse (VG Sira 9) après équilibration avec du $\mathrm{CO}^{2}$ suivant la technique classique d'EPSTEIN et MAYEDA (1953). Elle est exprimée en parts pour mille (ou $\delta$ ) par rapport au standard international SMOW (Standard Mean Ocean Water).

$$
\delta=\left(\left(R_{\text {Echant }}-R_{\text {standard }}\right)-1\right) 10^{3}
$$

avec $R={ }^{18} \mathrm{O} /{ }^{16} \mathrm{O}$

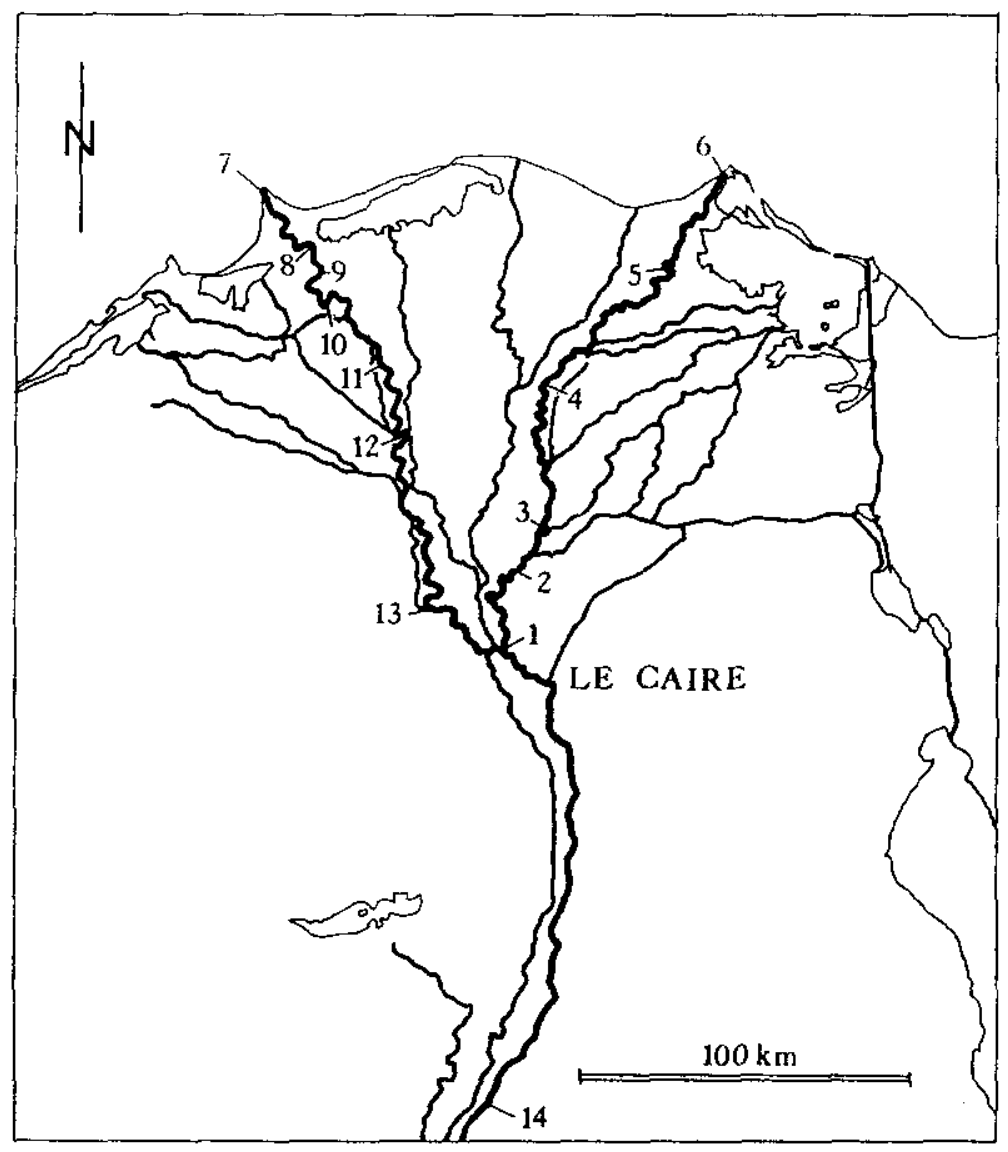

Figure 2.- Localisation des points d'échantillonnage du Nil le $12 / 08 / 84$.

Figure 2.- Situation of Nile water sampling stations, August 8, 1984. 
La précision est de $0,15 \%$. Les résultats bruts ainsi obtenus n'ont fait l'objet d'aucune correction liée à la salinité des eaux car celleci est mineure pour les eaux chloruxées sodiques comme c'est le cas ici (SOFER et GAT, 1972).

\section{2 - Le Nil et son delta (figure 2 et 3 )}

L'abondance en oxygène-18 en août 1984 est d'un peu plus de 3 \% ã Beni Suef (station 14) pour une teneur en $\mathrm{Cl}^{-}$très faible (entre 0,1 et $0,2 \mathrm{~g} \cdot \mathrm{d}^{-1}$ ). Les teneurs se maintiennent sensiblement dans le delta où cependant l'abondance en oxygène-18 s'élève globalement mais apparaft surtout plus variable dans l'espace. Ces phénomènes sont évidemment en rapport avec l'existence d'un réseau anastomosé de canaux d'irrigation dans tout le delta.

Au voisinage de la Méditerranée, l'abondance en oxygène-18 s'abaisse rapidement tandis que la salinitế augmente : celle-ci atteint même des valeurs supérieures à celles de la mex, sans doute à cause de contamination par les eaux des lagunes et des sebkhas qui s'étendent à la frange septentrionale du delta.

Ainsi, dans le système deltaique du Nil, à cause de la position géographique très particulièxe de ce fleuve, les eaux les plus salées sont aussi les plus pauvres en isotopes lourds. Voilà un phénomène qu'il est bon de garder à l'esprit lorsque l'on étudie le contenu isotopique de séries sédimentaires déposées sous climat aride, notament de dépôts paraliques.

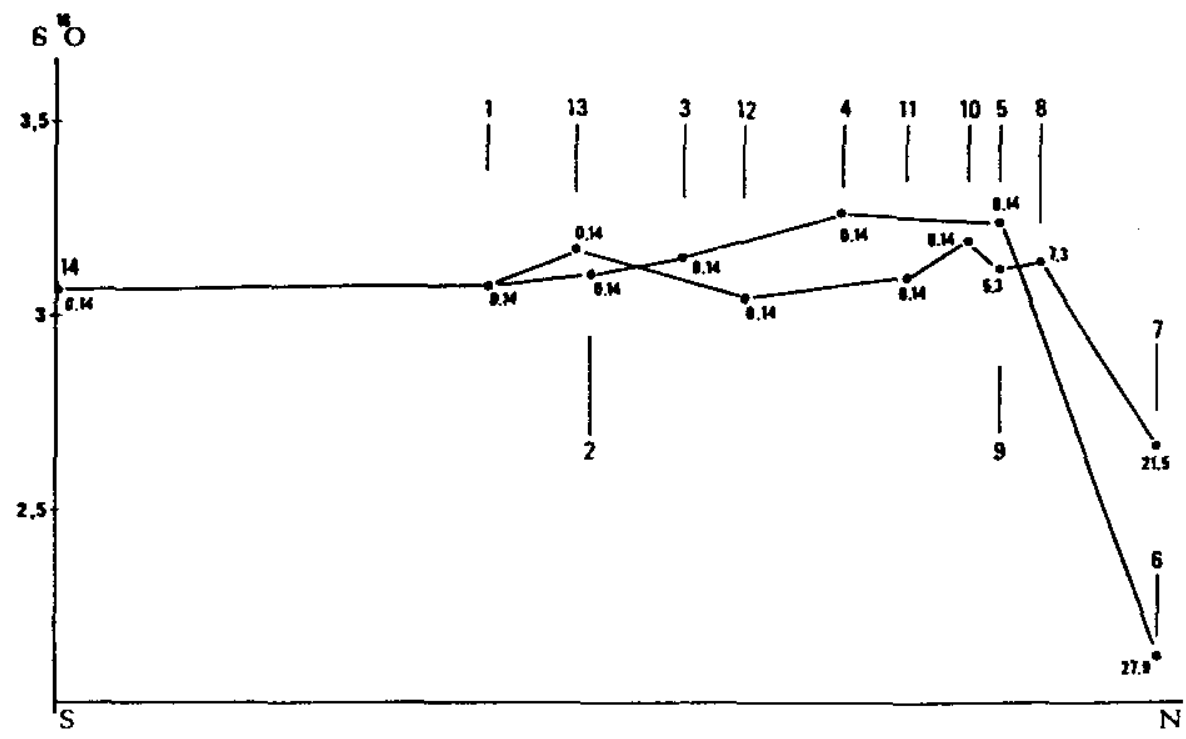

Figure 3,- Teneurs en oxygène-18 $(\delta \%)$ et en $\mathrm{Cl}^{-1}\left(\mathrm{~g} . \mathrm{I}^{-1}\right)$ des eaux du Nil le 12/08/84.

Figure 3.- 18-oxygen $(\delta \%)$ and $\mathrm{Cl}^{-}\left(\mathrm{g} . \mathrm{I}^{-1}\right)$ contents of Nile River water, august 8, 1984. 


\section{3 - Le Lac Temsah (figure 4 et 6 )}

L'alimentation mixte du bassin, par le canal de Suez qui y apporte des eaux salées d'origine marine d'une part, et par un bras du Nil d'autre part, induit en toute saison une diminution de l'abondance en oxygène-18 à partir du débouché du $\mathrm{Nil}$ vers la zone centrale du bassin.

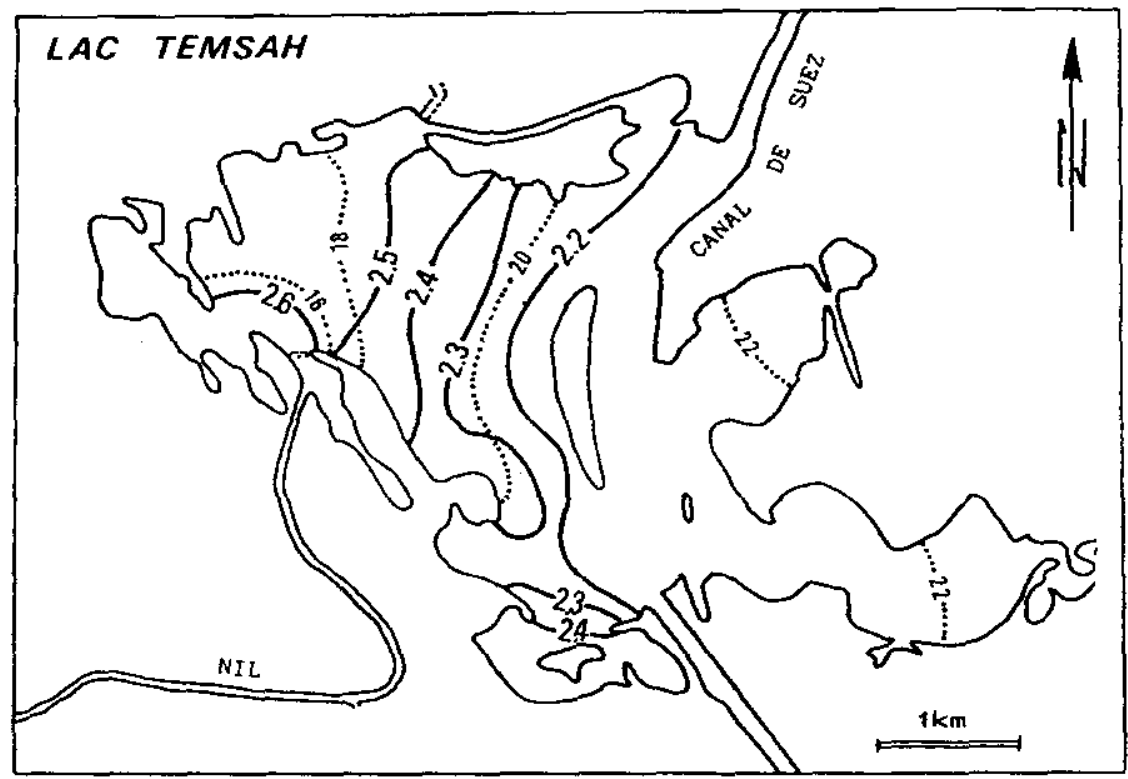

Figure 4.- Teneurs en oxygène-18 (traits pleins) et en $\mathrm{Cl}^{-}$(traits pointillés) des eaux de surface du Iac Temsah. Mars 1984.

Figure 4.- 18-oxygen (Continuous lines) and $\mathrm{Cl}^{-}$ (Dotted lines) contents of surface waters in Temsah Lake. March 1984.

A partir de celle-ci, la teneur en oxygène-18 remonte légèrement dans les baies marginales peu profondes oũ l'évaporation s'exerce sur une tranche d'eau faible. Ce phenomène est bien net en mars 1984 .

Le bassin présente une variation saisonnière marquée qui se traduit par une augmentation d'envixon $0,6 \%$ des teneurs en oxygène-18 de mars à aoot 1984. Si 1 'on compare le comportement isotopíque avec celui de la salinité, on constate que les eaux les plus riches en isotopes lourds sont soit des eaux dessalées par les apports continentaux soit des eaux légèxement sursalées par évaporation dans les bajes marginales peu profondes : dans un tel bassin, il ne peut y avoir de "signature isotopique" de la salinité, encore moins d'une éventuelle sursalure du milieu. 


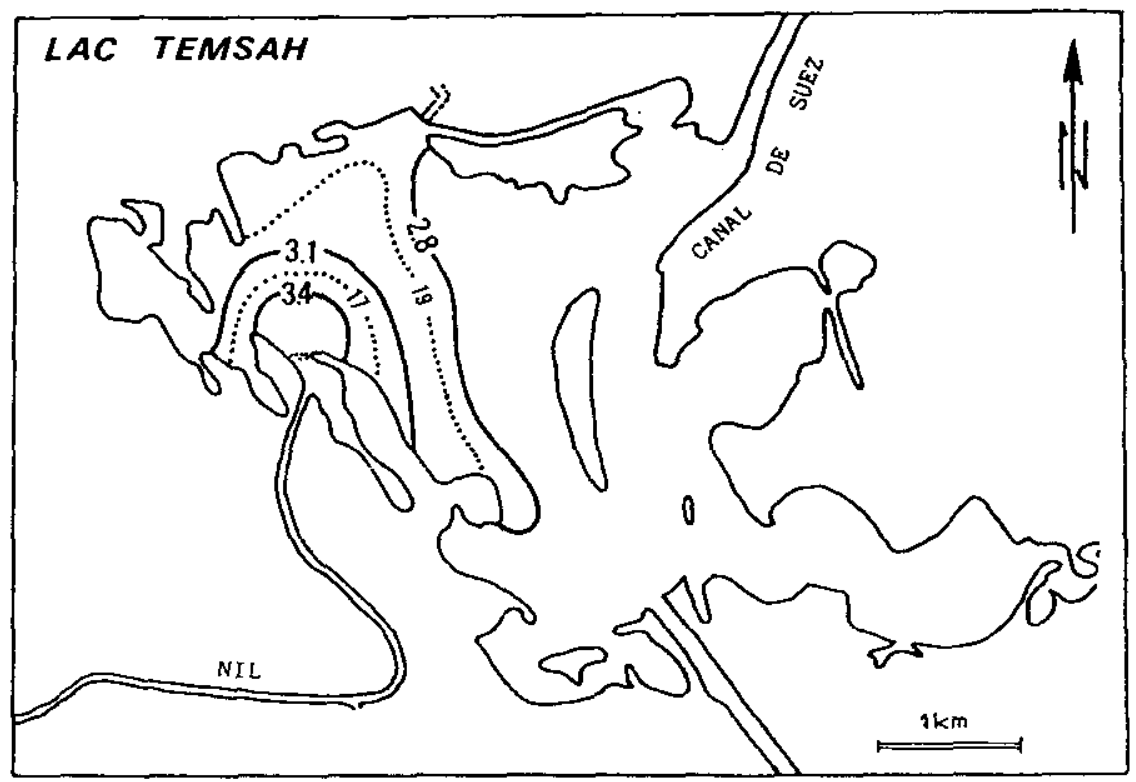

Figure 5.- Teneurs en oxygène-18 (traits pleins) et en $\mathrm{Cl}^{-}$(txaits pointilles) des eaux de surface du Lac Temsah. Aodt 1984.

Figure 5.- 18-oxygen (Continuous lines) and $\mathrm{Cl}^{-}$ (Dotted lines) contents of surface waters in Temsah Lake. August 1984.

4 - La lagune de Guemsah (figure 6 et 7)

La distribution de la teneur en oxygène-18 des eaux de surface dans la lagune de Guemsah dépend principalement de la circulation de cellesci.

En régime de vent du $\mathbf{N}$ (aoot 1984), l'alimentation du bassin se fait principalement par les passes so à proximité desquelles l'abondance en oxygène-18 est faible; celle-ci augmente vers le NE et se trouve maximale dans $I^{\prime}$ appendice confiné situé au $\mathrm{N}$ du bassin et sur le platier so qui est peut-être contaminé par les saumures de la Sebkha située à l'extrémité de la lagune.

Ces gradients de la composition isotopique se calquent assez bien sur ceux de la salinité.

Enfin, la variation saisonnière est bien marquée : elle influe sur la teneur en isotopes lourds des eaux entrantes mais aussi sur l'efficacité de l'évaporation sur le bassin lui-méme. Il en résulte des gradients plus prononcés en etté qu'en hiver. 


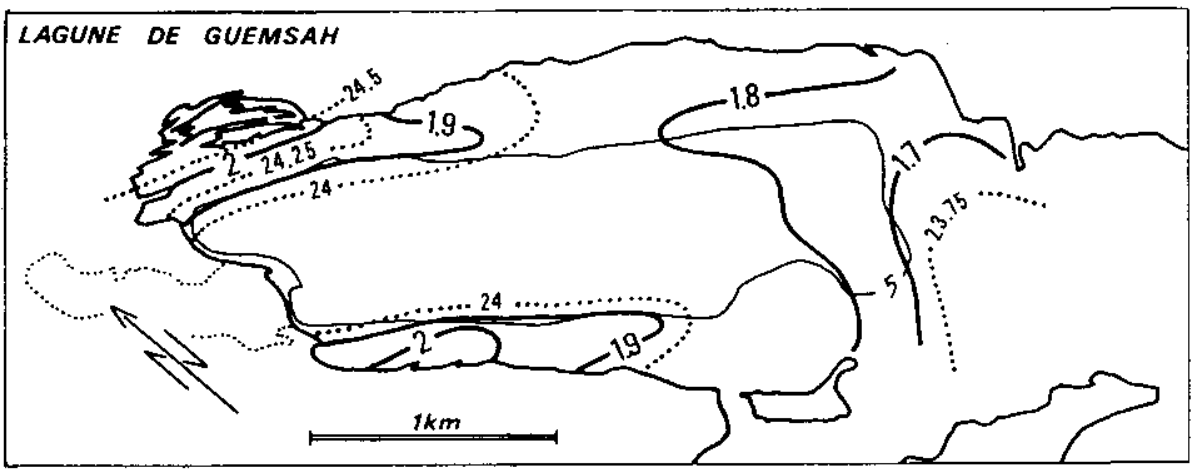

Figure 6.- Teneurs en oxygène-18 (traits pleins) et en $\mathrm{Cl}^{-}$(traits pointillés) des eaux de surface de la lagune de Guemsah. Janvier 1984.

Figure 6.- 18-oxygen (Continuous lines) and $\mathrm{Cl}^{-}$ (Dotted lines) contents of surface waters in Guemsah Lagoon. January 1984.

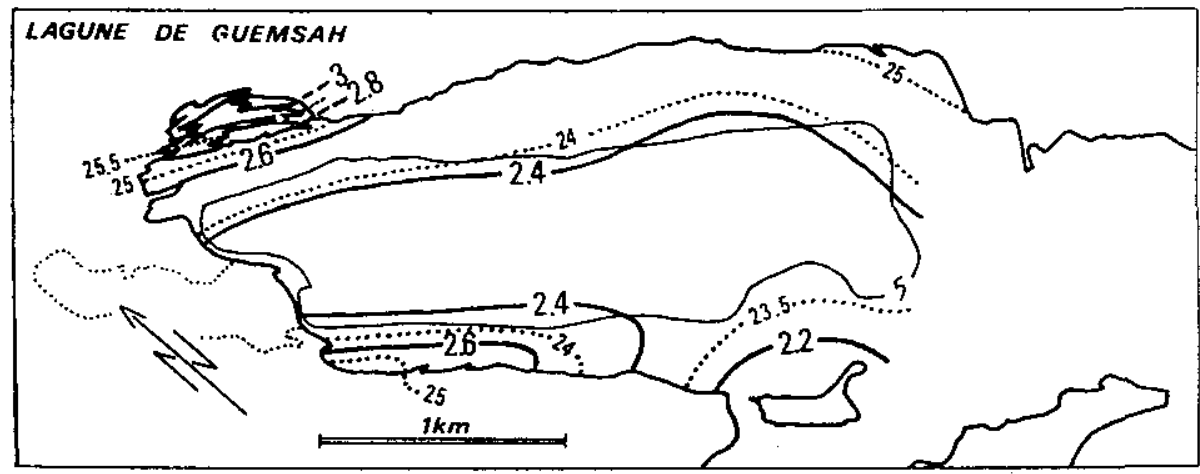

Figure 7.- Teneurs en oxygène-18 (traits pleins) et en $\mathrm{Cl}^{-}$(traits pointillés) des eaux de surface de la lagune de Guemsah. Aoat 1984.

Figure 7. - 18-oxygen (Continuous lines) and $\mathrm{Cl}^{-}$ (Dotted lines) contents of surface waters in Guemsah Lagoon. August 1984.

\section{5 - La Baie de Zeit (figure 8 et 9 )}

Le schéma qu'offre la Baie de zeit est plus simple puisqu'il n'y a qu'une seule passe. Le corps d'eau central sous influence marine directe comporte les eaux les moins. évaporées et les moins concentrées tandis que les platiers périphériques supportent des eaux enrichies en isotopes lourds et plus concentrées.

La variation saisonnière est encore marquée majs le régime des vents semble aussi jouer un rôle important. En janvier 1984 (par vent du S), 
la teneur en oxygène-18 et la salinité sont très uniformes sur le corps d'eau central ce qui indique un bon renouvellement des eaux. En aout 1984 (par vent du N) au contraire, les gradients sont beaucoup mieux marqués, ce qui tendrait à indiquer que le renouvellement des eaux est moins bon, en d'autres termes, que le confinement global du bassin est plus élevé.

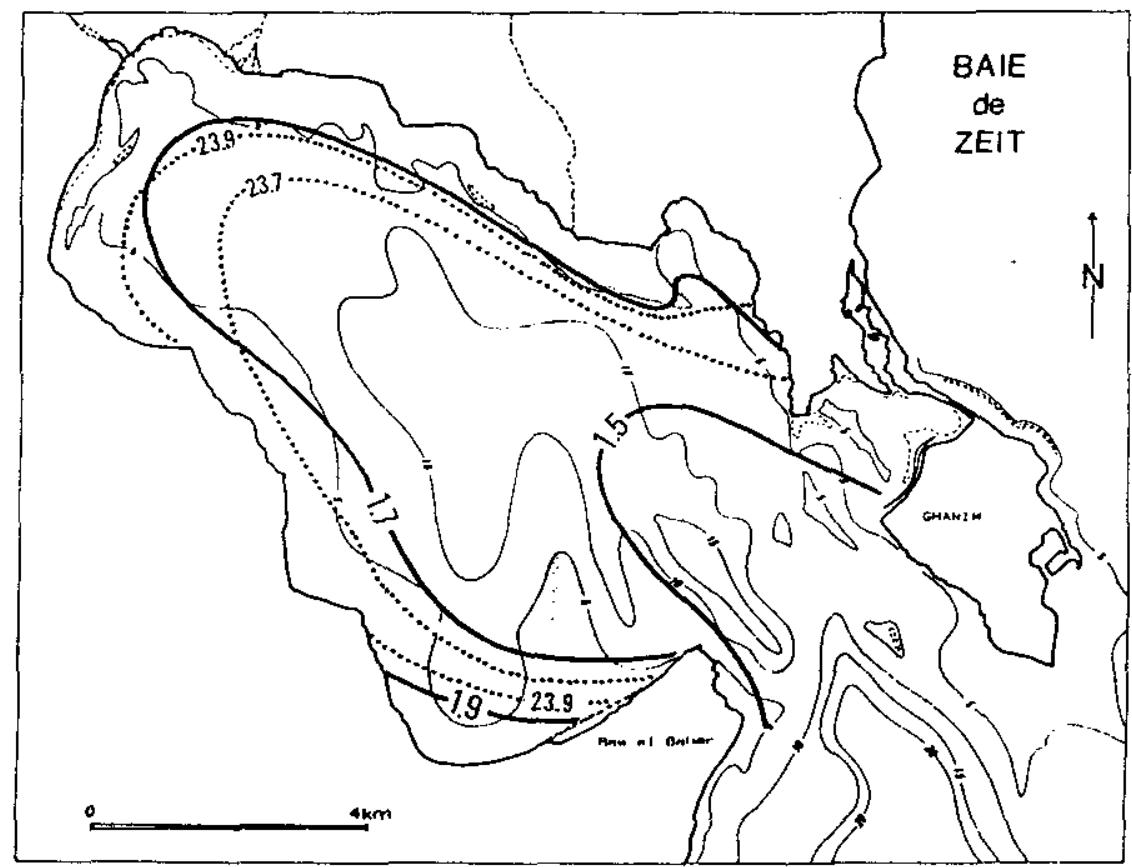

Figure 8. - Teneurs en oxygène-18 (traits pleins) et en $\mathrm{Cl}^{-}$(traits pointillés) des eaux de surface de la Baie de Zeit. Janvier 1984.

Figure 8.- 18-oxygen (Continuous lines) and $\mathrm{Cl}^{-}$ (Dotted lines) contents of surface waters in Zeit Bay. January 1984.

\section{6 - Les "Flaques" de la Sebkha Melaha}

I'eau qui alimente ce petit groupe de flaques et qui sourd en bordure de l'une d'elles n'est pas enrichie en isotopes lourds par rapport à la mer mais est plus chargée en sels : elle est peut-être contaminée par les saumures de la sebkha mais plus probablement a lessivé un peu de halite au cours de son trajet en sous-écoulement, donc a l'abri de l'évaporation, depuis la mer. En effet, les cailloutis qui constituent le cordon littoral sont localement cimentés par de la halite.

A partir du point d'alimentation, l'abondance en oxygène-18 crostt avec la teneur en chlorure de façon semblable à ce qui se passe dans les premiers stades de l'évaporation dans un marais salant (PIERRE et FONTES, 1982). 


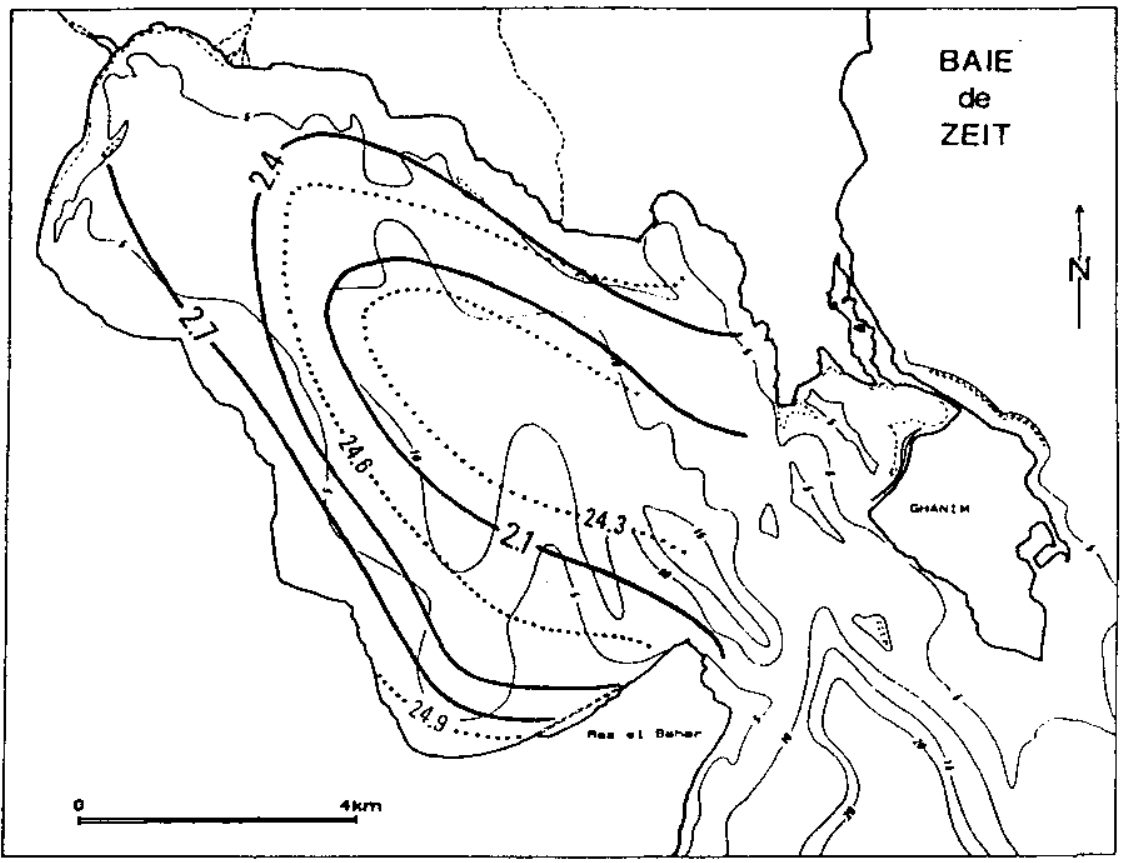

Figure 9.- Teneurs en oxygène-18 (traits pleins) et en $\mathrm{Cl}^{-}$(traits pointillés) des eaux de surface de la Baie de zeit. Aoút 1984.

Figure 9.- 18-oxygen (Continuous lines) and $\mathrm{Cl}^{-}$ (Dotted lines) contents of surface waters in zeit Bay, August 1984.

\section{7 - La Birket Karoun ou Lac du Fayoum (figure 10 et 11)}

Ici encore, on retrouve une variation saisonnière avec des valeurs de I'abondance en oxygène-18 plus élevées en aout qu'en mars de $0,5 \%$ environ, alors que la concentration moyenne en chlorure reste à peu près constante. Or pendant le meme temps, le niveau du lac s'est abaissé de $1,5 \mathrm{~m}$, ce qui est du même ordre de grandeur que la tranche d'eau évaporée sur la mëme période et correspond à environ $2 / 8$ du volume total de bassin. Si seule l'évaporation était responsable de cet abaissement du niveau, c'est-à-dire en l'absence de fuite, on devrait assister a une augmentation de la concentration en chlorure de 1 'ordre de $4 \mathrm{~g} \mathrm{l}^{-1}$, ce qui n'est pas le cas. De surcroit, à long terme, 1'accumulation des sels aboutirait au dépót de sédiments évaporitiques : on n'en trouve aucune trace. On peut donc affirmer que le bassin comporte des fuites comne l'écrivit naguère HERODOTE (vers 430 av. J.C). Ceci explique évidemment la relativement faible variation saisonnière de la teneur en oxygène-18.

En l'absence totale de données sur la composition saline et isotopique des apports, il est impossible de quantifiex de manière précise le flux des apports non plus que le flux des pertes par infiltration qui par ailleurs ont peu de chances de rester constants. Nous disposons cependant de données sur une plus longue période : d'après ISHAK et al., 
(1982), la salinité du lac en 1971-1972 s'étalait entre 28,6\% \% et entre $34 \%$ et $38 \%$ en 1978 , soit une augmentation de $6 \mathrm{g.1} \mathrm{I}^{-1}$ sur 7 ans.

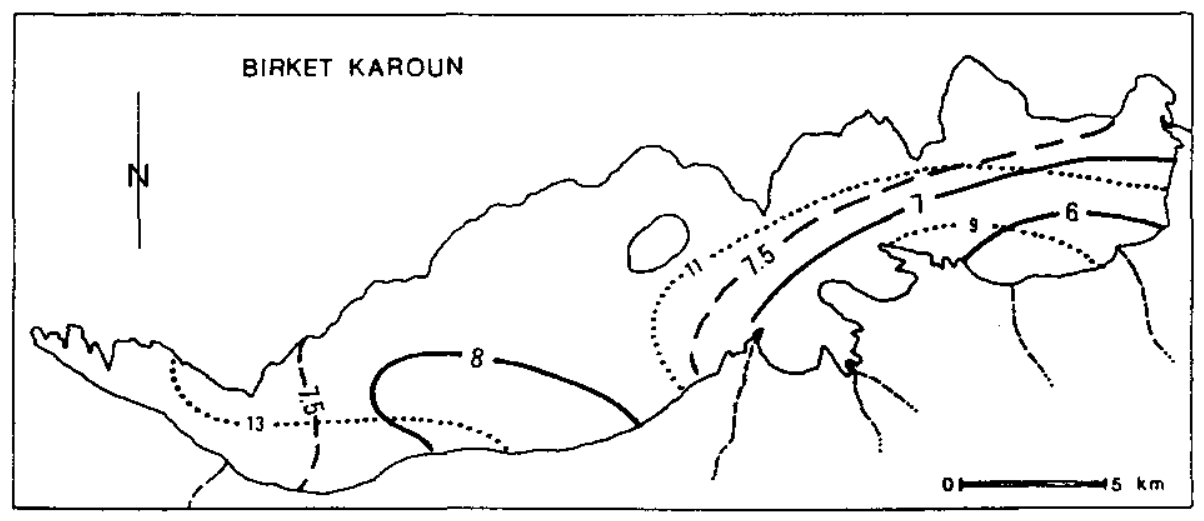

Figure 10.- Teneurs en oxygène-18 (traits pleins) et en $\mathrm{Cl}^{-}$(traits pointillés) des eaux de surface de la Birket Karoun. Maxs 1984.

Figure 10.- 18-oxygen (Continuous lines) and $\mathrm{Cl}^{-}$ (Dotted lines) contents of surface waters in Qamon Lake. March 1984.

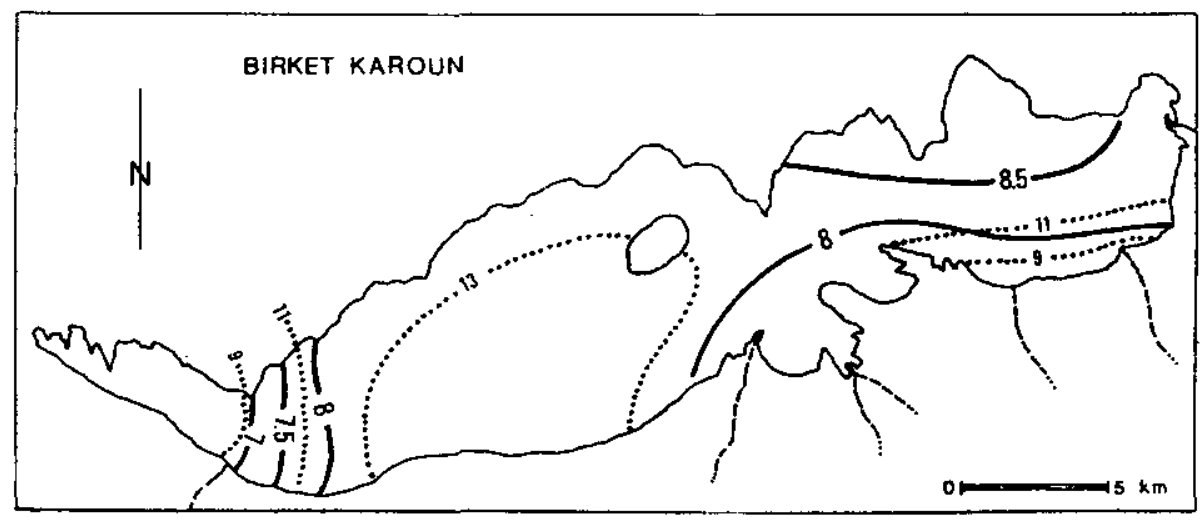

Figure 11.- Teneurs en oxygène-18 (traits pleins) et en $\mathrm{Cl}^{-}$(traits pointillés) des :eaux de surface de la Birket Karoun. Aout 1984.

Figure 11.- 18-oxygen (Continuous lines) and $\mathrm{Cl}^{-}$ (Dotted lines) contents of surface waters in Qarun Lake. August 1984.

En admettant que ces chiffres soient réellement représentatifs et que leurs moyennes soient significatives, il est possible d'estimer le bilan des sels dans le bassin en fonction des flux annuels respec- 
tifs des apports d'eau (A), de I'évaporation (E) et de I'infiltration (I).

$$
A=E+I
$$

$E$ est de l'ordre de grandeur du volume $V$ du bassin soit $800,10^{6} \mathrm{~m}^{3}$. En raisonnant à volume constant pour la période considérée, le bilan des sels dans le bassin s'écrit :

$$
7 A S_{A}+V S_{1972}=\sum I S+V S_{1978}
$$

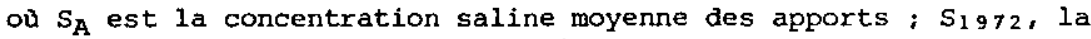
concentration moyenne du lac en $1972 ; S_{1978}$, la concentration moyenne du lac en 1978 ; S la concentration moyenne annuelle du lac de 1972 à 1978.

$$
7(I+V) S_{A}+V S_{1972}=I \sum S+V S_{1978}
$$

a'où :

$$
I=\frac{800.10^{6}\left(6-7 S_{A}\right)}{7 S_{A}-231}
$$

où les concentrations sont exprimées en $\mathrm{g} \cdot 1^{-1}$ et le flux en $\mathrm{m}^{3}$.an $\mathrm{n}^{-1}$.

Dans ces conditions, la concentration moyenne des apports est nécessairement supérieure à $0,86 \mathrm{~g} \cdot \mathrm{I}^{-1}$ et inférieure à $33 \mathrm{~g} \cdot \mathrm{I}^{-1}$. Dans cette fourchette, toutes les possibilités existent théoriquement. Poux fixer les idées supposons que le concentration moyenne des apports soit de $2 \mathrm{~g} \cdot \mathrm{1}^{-1}$; on obtient alors une perte par infiltration de $7,5.10^{6} \mathrm{~m}^{3} \cdot \mathrm{an}^{-1}$.

Pour une alinentation de $3 \mathrm{~g} \cdot \mathrm{1}^{-1}$, la perte serait de $56.10^{6} \mathrm{~m}^{3} \cdot \mathrm{an}^{-1}$. Ces chiffres ne paraissent pas excessifs vis-à-vis du volume total du bassin et des apports hydriques à celui-ci.

Dans la Birket karoun, la configuration des courbes d'isovaleurs de 1 'abondance en oxygène-18 souligne, en mars comme eri août 1984, 1 'existence des zones d'alimentation directes aux deux extrémités de la rive Sud du bassin ainsi que d'une alimentation diffuse par écoulement des nappes superficielles bordières le long de cette même rive. Rappelons que l'essentiel de l'alimentation du bassin s'effectue par la collecte des canaux d'irrigation et par déversement des nappes alluviales de la dépression du Fayoum qui sont en connexion avec celles de la vallée du Nil.

La répartition des valeurs isotopiques est en assez bonne conformité avec celle de la teneur en chlorure. En ce qui concerne le comportement géochimique et isotopique des eaux de bassins continentaux ou paraliques en climat semi-aride ou aride, les travaux de GONFIANTINI (1965), LLOYD (1966), FONTES et GONFIANTINI (1967), GAT et LEVY (1978), FONTES et al., (1979), McKENZIE et al., (1980) montrent que l'étude des variations des deux paramètres simples que sont la teneur en chlorure et 1'abondance en oxygène-18 apporte une contribution importante à la compréhension du fonctionnement de tels bassins. Récemment, MEDHIOUB et JUSSERAND (1984) ont ainsi pu expliquer en partie les relations hydrologiques entre la Bahiret el Biban (Sud tunisien) et ses sebkhas annexes. Dans le cas de 
la Birket karoun, en l'absence de données numériques sur les apports au réservoir (quantité, teneur en isotopes lourds) ainsi que sur les conditions évaporatoires (température, humidité de l'air, vitesse des vents, composition isotopique de la vapeur d'eau atmosphérique qui s'échange avec le liquide), il n'est pas possible d'appliquer les modèles classiques isotopiques d'évaporation (JAUZEIN, 1982 ; GILATH et GONFIANTINI, 1983). Cependant, moyennant quelques approximations et en raisonnant à long terme, il est possible de proposer un modèle simple en prenant en compte les bilans de masse en eau, en sels et en isotopes. Sachant qu'à long terme le lac Karoun est sensiblement en équilibre dynamique entre les apports, l'évaporation et l'infiltration, on peut écrire les bilans moyens suivants :

- bilan hydrique :

$$
\frac{d L}{d t}=A-E-I=0
$$

- bilan isotopique :

$$
d\left(L \delta_{L}\right)=d A \delta_{A}-d I \delta_{L}-d E\left(\delta_{L}+\varepsilon\right)=0
$$

avec $\mathrm{A}$ : volume des apports, $E$ : volume de I'évaporation, $\delta_{\mathbf{A}}:$ composition isotopique moyenne des apports, $\delta_{L}$ : composition isotopique moyenne du lac. $\delta_{L}+\varepsilon$ est la composition isotopique de l'eau qui s'évapore, $\varepsilon$ étant le coefficient de fractionnement isotopique global effectif qui est fonction des différents paramètres climatiques (température, humidité relative de l'air, vitesse du vent, etc.) et de l'activité de l'eau.

On notera que $\delta_{A}$ et $\delta_{L}$ sont des fonctions périodiques du temps (effet saisonnier) et que $E$ varie avec la surface du lac donc avec son volume $L$.

On introduit alors les salinités $s$ en supposant encore que le bilan hydrique du lac correspond à un équilibre dynamique à long terme et qu'il n'y a ni dépót ni dissolution de sels anciens à l'intérieur du lac lui-méme :

$$
d\left(L S_{L}\right)=d\left(A S_{A}\right)-d\left(I S_{L}\right)
$$

en simplifiant, on a, à l'état stationnaire :

$$
\begin{aligned}
& A=E+I \\
& A \delta_{A}=I \delta_{L}+E\left(\delta_{L}+E\right) \\
& A S_{A}=I S_{L}
\end{aligned}
$$

Avec ces trois équations à trois inconnues, si l'on connaf̣t $\delta_{A}, s_{A}$, $\delta_{\mathrm{L}}$ et $\varepsilon$, on peut déterminer le rapport des flux et on en tire :

$$
\varepsilon=\frac{S_{L}\left(\delta_{L}-\delta_{A}\right)}{S_{A}-S_{L}}
$$

En faisant varier $\varepsilon$ (effectif), on peut en déduire les couples de valeurs $\delta_{A}$ et $S_{A}$ compatibles entre elles. Or, $\varepsilon$ est la somme du facteur d'enrichissement isotopique à $l$ 'équilibre $\left(\varepsilon_{\mathrm{e}}\right)$ et du facteur d'enrichis- 
sement cinétique $\left(\varepsilon_{k}\right)$ :

$$
E=\varepsilon_{e}+\varepsilon_{k}
$$

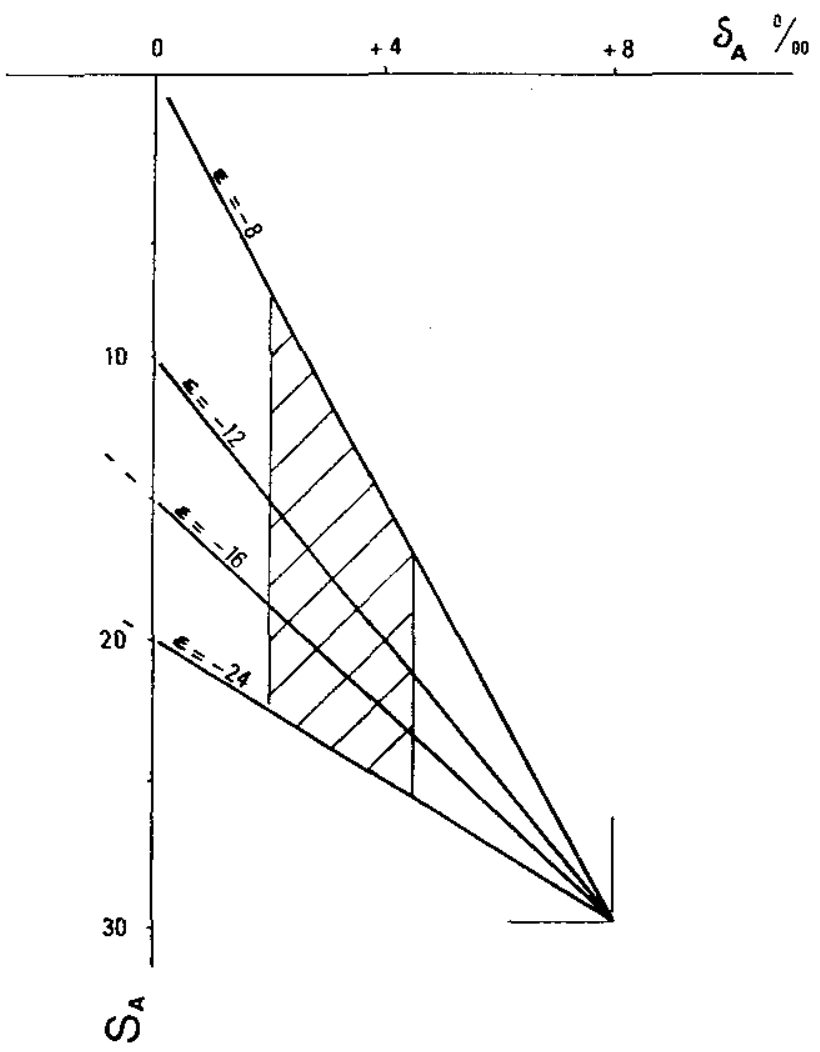

Figure 12.- Valeurs de $\delta_{A}$ et de $S_{A}$ en fonction de $\varepsilon$. Zone hachurée : zone des valeurs vraisemblables.

Figure 12.- Values of $\delta_{A}$ and $S_{A}$ as a function of $\varepsilon$. Hatched zone : probable values.

Sous climat axide ou semi-aride et pour des températures comprises entre $25^{\circ} \mathrm{C}$ et $30^{\circ} \mathrm{C}, \varepsilon_{\mathrm{e}}$ ne peut étre supérieux à $-8 \%$. $\varepsilon_{\mathrm{k}}$ dêpend surtout des conditions aérodynamiques du milieu et augmente avec la turbulence de l'air : en régime turbulent, $\varepsilon_{\mathrm{k}}$ est supérieur à $-14 \%$. Ainsi, on peut faire varier $E$ entre $-8 \%$ et $-24 \%$ en admettant un régime généralement. turbulent (vents forts du secteur . N, mouvements de convexion). La figure 12 indique les couples de valeurs $\delta_{A}$ et $S_{A}$ compa tibles entre elles pour ces valeurs de $E$ et pour une composition

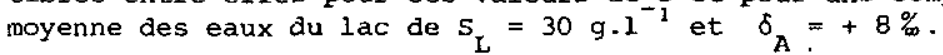

En se donnant pour la composition isotopique moyenne des apports un intervalle de $+2 \%$ a $+4,5 \%$ (d'après sIMpson et al., (1987), la composition isotopique en oxygène-18 des eaux du paramètre irrigué du delta du Nil varie entre $+4 \%$ et $-6 \%$, on obtient des valeurs de la concentration saline moyenne des apports comprise entre $7,5 \mathrm{~g} \cdot \mathrm{I}^{-1}$ et $26 \mathrm{~g} \cdot \mathrm{l}^{-1}$, 
les valeurs les plus vraisemblables se situant aux alentours de $20 \mathrm{~g} \cdot \mathrm{I}^{-1}$.

De telles concentrations salines ne peuvent guère s'expliquer que par un lessivage de sels sur le parcours des eaux d'alimentation de la Birket; elles impliquent aussi des valeurs du flux d'infiltration, c'est-à-dire des pertes, très élevées, de l'ordre de grandeur du volume du bassin ( $c f$. supra). Ainsi, la Birket Karoun apparaft plus comme une fenêtxe dans la nappe nilotique que comme un bassin endoréique, ce qui ne saurait étonner au vu de la pluviométrie annuelle.

Enfin, I'existence de fuites, surtout de l'ampleur que suggèrent les considérations précédentes, interdit d'envisager une quelconque alimentation marine du bassin actuel. En outre, même si une telle alimentation avaj.t existé à une époque ancienne du Quaternaire, ce qui reste très douteux (IBRAHIM, 1986), il ne pourrait subsister dans le bassin qu'une fraction infinitésimale d'éléments d'origine marine. La Birket Karoun est donc un bassin strictement continental mais c'est peut-être le lessivage de séries marines anciennes (pliocènes ?) qui y maintient un milieu compatible avec la vie d'organismes généralement inféodés au domaine marin.

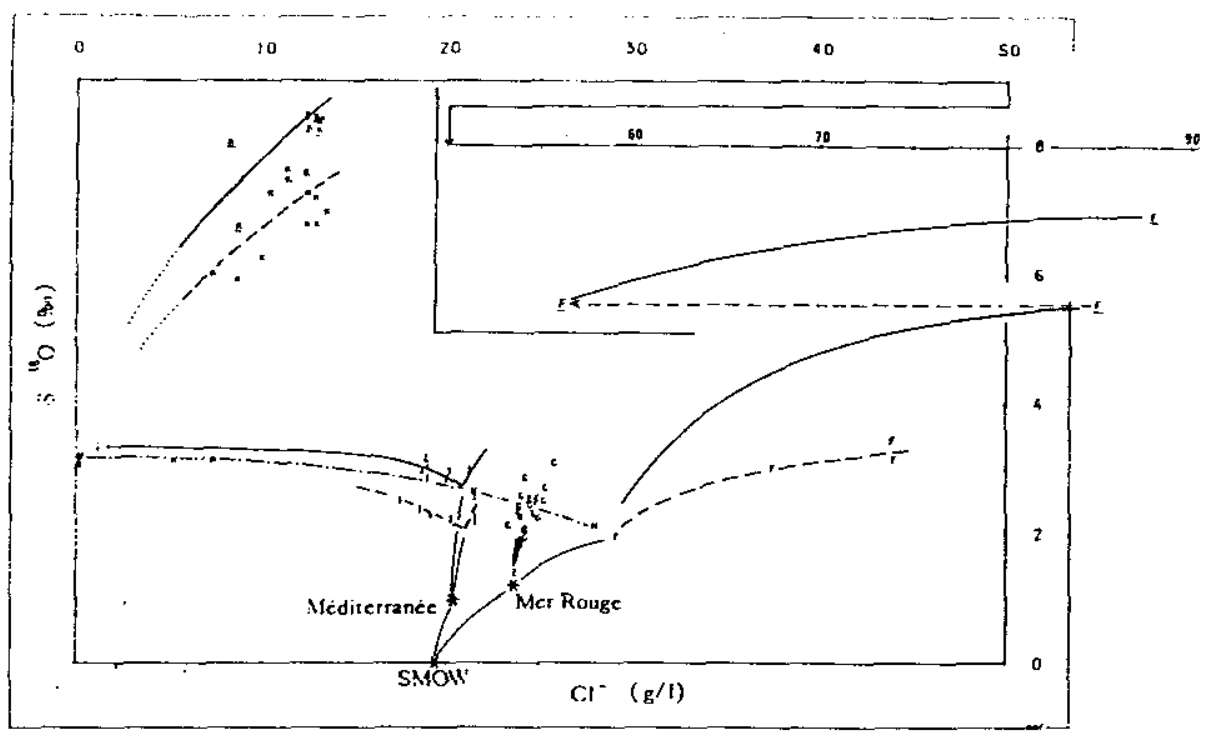

Figure 13.- Relations entre les teneurs en oxygène-18 et les teneurs en chlorure des eaux des bassins êtudiés.

Figure 13.- 18-oxygen vs $\mathrm{Cl}^{-}$. in surface waters of studied basins.

Lignes pleines : été. Lignes tiretées : hiver.

$N$ : eaux du Nil ; G : lagune de Guemsah ; Z : Baie de Zeit ;

$F$ : "Flaques" de la Sebkha Melaha; I : Lac Temsah ; $K$ : Birket Karaun.

Dashed lines : winter. Solid lines : summer.

$N$ : Nile River; $G$ : Gemsah Lagoon; $Z$ : Zeit Bay; $F$ : Sabkha Malaha ponds ; I : Temsah Lake; K : Qarun Lake. 


\section{3 - LES RELATIONS ENTRE LA CONCENTRATION EN CHLORURE ET L'ABONDANCE EN OXYGËE-18 DANS LES BASSINS NATURELS ÉGYPTIENS ÉTUDIÉS}

Les résultats des analyses chimiques et isotopiques obtenues ont été reportés sur un diagramme où les axes représentent la concentration en chlorure d'une part, 1'abondance en oxygène-18 ( $\delta$ ) d'autre part (figure 13 et 14 ). Ce diagramme montre clairement la variation isotopique saisonnière de chaque bassin qui est de $0,5 \%$ en moyenne sauf pour les "Flaques" de la Sebkha Melaha où elle est mieux marquée.

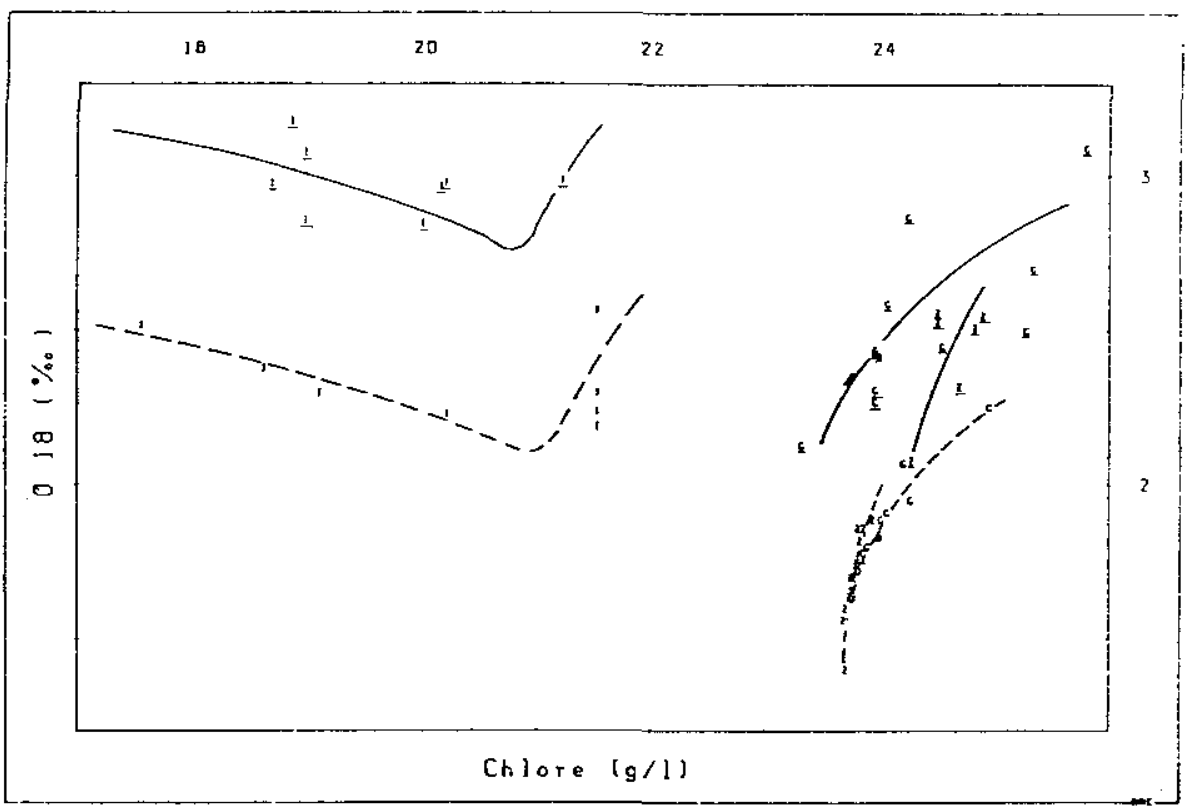

Figure 14.- Détail de la figure 13.

Figure 14.- Zoom on part of figure 13.

Dans les bassins à alimentation unique (Lagune de Guemsah, Baie de zeit, "Flaques", Birket Karoun) les courbes que 1 'on peut tracer montrent que la relation entre les deux paramètres est grossièrement logarithmique. Pour le Lac Tensah, à alimentation mixte, les courbes obtenues notamment pour mäs 1984 comportent deux branches ; I'une représente l'évolution évaporitique dans la partie orientale du bassin, l'autre, le mélange, dans sa partie occidentale, des eaux d'origine marine et des apports continentaux surêvaporés du système nilotique. On notera la similitude de cette branche de courbe avec la courbe qui correspond au delta du Nil.

Ce diagramme montre encore un phénomène assez remarquable. En dépit de la variation saisonnière globale de chaque système, les courbes 
représentatives ont sensiblement la même allure pour l'hivex que pour l'été. Ceci est particulièrement net pour la lagune de Guemsah et pour la Baie de zeit et signifie que dans le cas de bassins à alimentation unique on pourrait définir un "facteur d'enxichissement isotopique global" propre à chaque bassin. Sa valeur dépend des caractéristiques de celui-ci au rang desquelles figurent probablement la surface, le rapport surface/volume, I'importance de la zone sous empxise du vent, la cixculation des eaux et des paramètres climatiques (température, humidité relative, régime aérodynamique, etc.).

Enfin, on remarquera que les points représentatifs des solutions naturelles étudiées sont plus dispersês en été qu'en hiver. Ceci est particulièrement net pour la lagune de Guemsah, la Baie de Zeit et le Lac Temsah. Il est probable qu'en été les gradients thermiques plus accentués entre la surface et le fond engendrent des mouvements de convexion plus ou moins complexes perturbant la trajectoire des différentes masses d'eau et favorisant les mélanges entre celles-ci.

\section{CONCLUSION}

A l'aide de cette etude, on montre clairement que les seules valeurs isotopiques ou les seules valeurs de la chlorinité demeurent insuffisan-

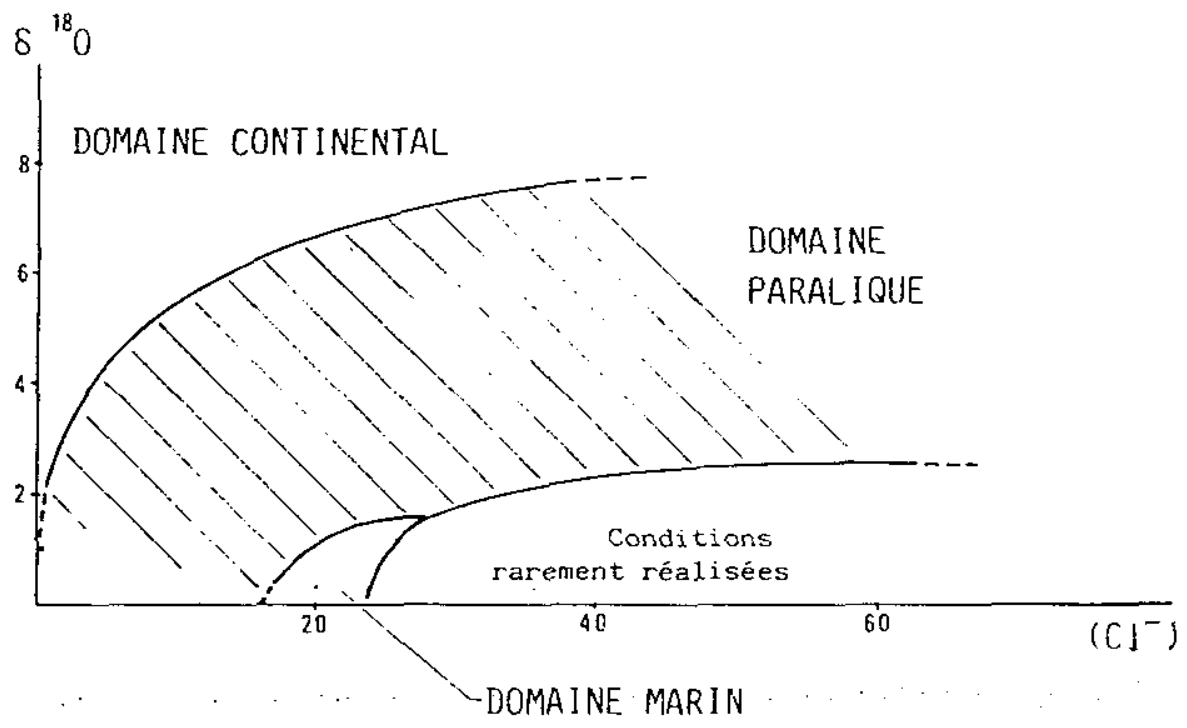

Eigure 15.- Essai de délimitation des eaux marines, paraliques et continentales, dans le contexte géographique et climatique égyptien à partir de leurs teneurs en oxygène-18 et en $\mathrm{Cl}^{-}$.

Figure 15. - Tentative delimitation of marine, paralic and continental waters, within the geographical and climatic eguptian environment from their 18-oxygen and chloride contents. 
tes, dans un tel contexte physiographique, pour définir le milieu concerné. Ainsi, une valeur de l'abondance en oxygène-18 de +3 w correspond aussi bien à un milieu continental (eau douce ou salée), qu'à un milieu paralique dessalé par des apports d'eau douce, qu'à un milieu paralique évaporitique. On peut seulement dire que le milieu a fort peu de chance d'etre marin.

Un point important de l'étude est l'absence de corrélation directe entre composition isotopique et teneur en chlorure des eaux dans les bassins à apports d'eaux continentales enrichies en isotopes lourds.

Si l'on associe les deux paramètres que sont la teneur en chlorure et 1 'abondance en oxygène-18, il devient possible de définir très grossièrement et dans le seul contexte géographique et climatique des zones arides, les valeurs de ces paramètres correspondant aux trois grands domaines de l'hydrosphère : le domaine marin, le domaine continental et le domaine paralique (figure 15).

Ces données de 1'Actuel, dans une ambiance géographique originale mais qui a pu exister au cours des temps géologiques, illustrent l'intêrêt des études isotopiques pour les reconstitutions paléogéographiques, surtout lorsqu'elles sont confrontées à d'autres paramètres géochimiques.

\section{REMERCI EMENTS}

Cette êtude a été réalisée, dans le cadxe de recherches sur l'organisation biogéologique de bassins paraliques égyptiens, grace à une convention octroyée par Total Compagnie Française des pétroles. Nous en remercions cette Compagnie et André Maurin, instigateur de ces travaux.

\section{RÉFÉRENCES BIBLIOGRAPHIQUES}

EPSTEIN S, et MAYEDA T.K. (1953). Variations of ${ }^{B} O$ content of waters from natural sources. Gecchim. Cosmachim. icta, 4: $213-224$.

FONTES J. Ch. et CONFIANTINI R. (1967), Comportement isotopique au cours de l'évaporation de deux bassins sahariens. Earth Planet. Sci. Lett., 3: 258-386.

FONTES J. Ch. FLORKOWSKI J., POUCHAN P. et ZUPPI U.M. (1979). Preliminary isotopes study of Lake Assal system (Republic of Djibouti). Isotopes in lake studies, I.A.E.A., Vienna, 163-174.

GAT J.R. et LEVY I. (1978). Isotope hydrolcogy of inland sabkhas in Bardawil area, Sinai, Oceanogr. Limnot., 23: 841.
GILATH Ch. et GONFIANTINI R. (1983). Lake dynamics. In : Guide-book on nuctear techniques in hydrolugy, I.A.E.A., Vienna, 129-161.

GONFIANTINI R: (1965). Effetti isotopici nell'evaporazione di acque salate. Atti Soc. Toscana Sci. Nat., Pisa, A, LXXII, 550-569.

GRIFFITHS J.F. (1972). Climates of Africa. In : World Survey of climatology, LANDSBERY .. Ed., 10, Elsevier, 456 p..

HERODOTE (vers 430 av. J.C.). Histoires. Trad, LEGRAND Ph. E. (1957), club Français du Livre, paris, 949 p.. 
IBRAHIM A. (1986). Contribution a l'etude biogéologique de bassins paraliques egyptiens. Th. Doct. Etat. Univ. Nantes, 252 P. .

IBRAHIM A., GUELORGET O., FRISONI G.F. ROUCHY J.M., MAURIN A. et PERTHUISOT J.P. (1985). Expressions hydrochimiques, biologiques et sédimentologiques des gradients de confinement dans la lagune de Guemsah (Golfe de Suez, Egypte). Oceanol. Acta., 8, 3, 303-320.

ISHAK M. M., ABDEL, MALEK S.A. et SHAFIK M.M. (1982). Development of mullet fisheries (Mugilidae) in Lake Qarun, Egypt, Aquaculture, $27: 251=260$.

JAUZEIN A. (1982). Deuterium et oxygene is dans les saumures. Modélisation et implications sédimentologiques. C.R. Acad. Sci., 11 (294): 663-668.

LLOYD R.M. (1966) . Oxygen isotope enrichment of seawater by evaporation. Geochim. Cosmochim. Acta, 30: 801 .

MCKENZIE J., HSU K.J. et SCHNEIDER J.F. (1980). Movements of subsurface waters under the Sabkha Abu Dhabi, U.A.E. and its relation to evaporative dolomite genesis. Soc. Ec.n. Paleo. Min., Spec. Pub. . 28: 11-30.

MEDHIOUB $\mathrm{K}$. et JUSSERAND $c$. (1984). Le comportement chimique et isotopique des eaux du complexe paralique de la dépression de Ben Guirden (SE tubnisien). Rev. Fr. Sci. Eau, 3(4): 335-358.

OMAR M.H, et EL BAKRY M.M. (1981). Estimation of evaporation from the Lake of Aswan high dam (Lake Nasser) based on the measurements over the lake. Agricul, meteorol., 23: 293-308.

PERTHUISOT J.P. et GUELORGET O. (1987a). Les milieux paraliques : diversité et unite. Bulz. Ecul., (sous-presse).

(1987b). Le confinement et 1 'organisation biogeologique des aires paraliques. Bull. IGBA, (sous-presse).

PIERRE C. et FONTES J. Ch. (1982). Etude isotopique des saumuxes et des gypses des marais salants de Salin-de-Giraud (Sud de la France). Geol. Medit., IX(4): 479-486.

SIMPSON H.J., HAMZA M., WHITE J., NADA A. et AWAD M. (1987). Evaporation enrichment of ${ }^{2} \mathrm{H}$ and ${ }^{10} \mathrm{O}$ in arid zone irriqation. Isotope iech. in Wats: $\mathrm{is}$. development, I.A.E.A., Vienna, 24i-250.

SOFER 2. et GAT J.R. (1972). Activities and concentration of 18-oxygen in concentrated aqueous solutions ; analytical and geophysical implications.

Earth Plonet. Sci. Letters., 15: 232-238. 